\title{
LITERATURBERICHT
}

\section{Neue Akteure, neue Prozesse - alles beim Alten? Über den schwierigen Umgang mit der Ambivalenz der arabischen Transformationsprozesse ${ }^{1}$}

Irene Weipert-Fenner

New Actors, New Processes - Nothing's Changed? About the Difficulties Dealing with the Ambivalence of the Arab World's Transformation Processes

Abstract: Three years after the mass protests in the MENA region it is still unclear if the transformations will lead to democracy. This article reflects the challenge that the openended developments posed for the first "wave" of publications. It starts with the difficulty to find an appropriate term for the events of 2011: "Arab Spring" or "Arab Revolutions"? Secondly, the re-evaluation of autocratic regime research highlighted the relevance of social movements as well as the interconnectedness of politics from above and below. Yet, more general conclusions could hardly be drawn with the transformations unfolding. Finally, studies on Egypt and Tunisia underlined the ambivalence by showing how the army, the Islamists and the media supported as well as threatened democratization; EU democracy promotion, however, seemed unable to adapt sufficiently to the developments.

Keywords: Arab spring, Middle East and North Africa, authoritarianism, democratization

Schlagwörter: Arabischer Frühling, Nordafrika und Naher Osten, Autoritarismus, Demokratisierung

\section{Einleitung}

Als 2011 die große Protestwelle in Nordafrika und dem Nahen Osten (MENA) Diktatoren zu Fall brachte, war die Euphorie groß. Demokratie schien endlich in die letzte, fast komplett autoritär regierte Region der Welt Einzug zu halten. Der Fokus auf die Stabilität autoritärer Regime, der die nahostbezogene Politikwissenschaft in den 2000er Jahren dominiert hatte, wurde in der Öffentlichkeit als passé deklariert. ${ }^{2}$ Heute, drei Jahre später, werden Stimmen lauter, die die Demokratisierung als stagniert, wenn nicht gar als gescheitert, betrachten. Die Aufstände von 2011 werden somit als Erschütterungen in den nun wieder stabilisierten Autokratien gesehen, die mit wenigen Modifikationen weiter existieren.

1 Ich danke André Bank, Thomas Richter und Mariam Salehi für ihre wertvollen Kommentare und Anregungen zu einem früheren Entwurf dieses Artikels.

2 Siehe die Kontroverse zwischen Adrian Lobe (2011) und Oliver Schlumberger (2011). 
In der Tat kann im Moment wohl niemand den Ausgang der Transformationsprozesse unterschiedlichster Art vorhersagen. Der vorliegende Literaturessay soll daher einen Überblick über die bisherigen Debatten geben, die die Entwicklungen seit 2011 zu verstehen suchen und die allesamt dadurch gekennzeichnet sind, dass sie in einem Spannungsfeld zur Ergebnisoffenheit der Prozesse stehen: Zunächst soll die Auseinandersetzung mit den am häufigsten verwendeten Begriffen wie „Arabischer Frühling“ und „Revolution“ dargestellt werden, die vor allem zeigt, dass diese stets Vorannahmen bezüglich des Verlaufs und des Ausgangs der Entwicklungen enthalten. In einem zweiten Schritt werden theoretische Neuüberlegungen, die die Proteste von 2011 ausgelöst haben, diskutiert. Diese drehen sich um die Fragen, wer die relevanten Akteure in einer Autokratie sind und wie Prozesse innerhalb des Regimes verlaufen. Aber auch hier ist eine eindeutige Bewertung schwer, wenn das Ergebnis der angestoßenen Entwicklungen - und damit auch die Bedeutung der Proteste von 2011 selbst - noch nicht klar ist. Die Schwierigkeit, dass die Transformationsprozesse nicht linear in eine Richtung Demokratie oder Autokratie - verlaufen, zeigen auch die Forschungsarbeiten zu den Fällen Tunesien und Ägypten, die im dritten Teil diskutiert werden. In den beiden Ländern, in denen es zum Sturz des Diktators gekommen war, wird anhand der bisherigen Analysen einzelner Themenfelder wie der Rolle der Armee, des politischen Islams und der Medien deutlich, wie ambivalent deren Rolle für die Demokratisierung sein kann. Schließlich wird die bisherige Einschätzung der Demokratieförderung der EU beleuchtet, die sich der Komplexität der Prozesse nicht angepasst hat und damit selbst ambivalent bezüglich demokratiefördernder oder -verhindernder Wirkung ist.

\section{Den Wandel in Worte fassen: Arabischer Frühling vs. Revolution}

Was ist im Jahr 2011 in der arabischen Welt passiert und wie lässt sich dies mit einem Begriff adäquat bezeichnen? Im westlichen Diskurs hat sich die Bezeichnung „Arabischer Frühling “ zunächst durchgesetzt, gefolgt in der angelsächsischen Literatur von „Arab Awakening“. In letzter Zeit sind „Arabische Revolte“, „Aufstand“ und „Arabellion“ beliebter geworden. In arabischsprachigen Veröffentlichungen ist dagegen „Revolution“ der Schlüsselbegriff, auch für die Fälle, in denen es nicht zum Sturz des Herrschaftsträgers kam. Sämtliche Begrifflichkeiten sind jedoch selbst Gegenstand der Auseinandersetzung, da sie einen bestimmten Rahmen für die Interpretation der weiterlaufenden Ereignisse setzen.

So wird der Begriff „Arabischer Frühling“ dafür kritisiert, den Eindruck der Kurzfristigkeit zu erwecken und damit die Erwartung zu schüren, dass Transformationen in wenigen Monaten erfolgen könnten. Damit reduziere er das Verständnis für nicht-lineare Verläufe (Perthes 2013, S. 50-51), bei denen Demokratie kein direktes Ergebnis der Massenproteste sein wird, und erkläre damit Demokratisierung schnell zum gescheiterten Projekt, was zu Wortspielereien wie „Arabischer Herbst“ oder gar „Arabischer Winter" führt (beispielsweise bei Žižek 2012). Dabei schwingt für Khouri mit, dass bei der Auswahl des Begriffs bereits das Scheitern angenommen werde (2011). Der politische Frühling bezeichne in der Geschichte immer nur erfolglose Versuche einer politischen Neuord- 
nung, wie den „Prager Frühling“, die gescheiterten Revolutionen in Europa 1848 oder die als „Damaszener Frühling“ in die Debatte eingegangenen politischen Liberalisierungsschritte von Bashar al-Assad 2001. Doch trotz der Kritik ist der Begriff „Frühling“ weit verbreitet und öffentlich positiv konnotiert. Dies macht ihn besonders bei der Wahl von Publikationstiteln beliebt, weswegen beispielsweise Haas und Lesch ihre Titelwahl explizit damit begründen (2012). Angesichts der zahlreichen Probleme, die mit dem Begriff zusammenhängen, erscheint es ratsam, ihn möglichst zu vermeiden, wie es der vorliegende Artikel unter Verwendung von Begriffen wie „Proteste“ und „Transformationen“ tut. Da der Terminus im öffentlichen Diskurs jedoch weiterhin dominant ist, bleibt es wichtig zu betonen, dass es sich hierbei nicht um die spontane Errichtung der Demokratie in der MENA-Region handelt, sondern um den Beginn komplexer und ambivalenter Transformationsprozesse, die zur Demokratie führen können, aber nicht müssen.

Doch auch das Attribut „arabisch“ wird kontrovers diskutiert. So werden „Arabischer Frühling“ und „Arabisches Erwachen“ aus einer postkolonialen Perspektive dafür kritisiert, das durch Stereotype geprägte Bild des Nahen Ostens als „des anderen“ fortzuführen (El-Mahdi 2011). Auf der anderen Seite, so Gause, sei eine pan-arabische Identität politisch relevant geworden, die die Menschen in arabischen Ländern dazu gebracht habe, sich gegenseitig zu Protesten zu inspirieren und zu ermutigen. Die Grüne Bewegung im Iran im Jahr 2009 hatte diesen Effekt nicht (Gause 2011, S. 87-89). Zudem sei das Auftreten einer Demokratisierungswelle in regionalen Clustern auch in anderen Teilen der Welt zu beobachten gewesen, da Demonstrations- und Diffusionseffekte durch geteilte Sprache und Kultur - wie in der MENA-Region gegeben - begünstigt würden (Grand 2011, S. 22).

Mit je nach Sprache sehr unterschiedlichen Konnotationen ist der ebenfalls geläufige Ausdruck „Arabisches Erwachen“ versehen. Von den Benutzern selbst wird er kaum reflektiert, wie zum Beispiel bei Pollack (2011). ${ }^{3}$ Auch Tariq Ramadan, islamischer Reformdenker, bedient sich dessen unkommentiert (2012). Von einer postkolonialen Perspektive hingegen wird der Begriff „Erwachen“ für die Anlehnung an eine Terminologie der Aufklärung und orientalistischer Stereotypen kritisiert, die einen schlafenden Orient im Gegensatz zu einem wachen, sich entwickelnden Okzident evozierten (Czajka 2011, Hudson 2011). Dies ist problematisch, da er suggeriert, dass es vor dem Jahr 2011 keine Entwicklungen gegeben habe. Sieht man den Begriff jedoch im geschichtlichen Kontext der MENARegion, so erinnert er an regionale Vorläufer von Reform und Revolution, zum Beispiel an die „nahda“, die oft synonym mit „awakening“ verwendet, aber gemeinhin als „Renaissance“ übersetzt wird. Dieser Sammelbegriff steht für eine politische, religiöse, sprachliche und literarische Erneuerungsbewegung im 19. und 20. Jahrhundert, die in Beirut, Damaskus und Kairo ihren Anfang nahm. Sie führte, auch in Auseinandersetzung mit den europäischen Kolonialmächten, zur Entwicklung des Nationalismus in den arabischen Ländern um die Zeit des Ersten Weltkriegs, was von George Antonius 1938 als „arabische[s] Erwachen“ bezeichnet wurde. Dawisha (2013), der das Jahr 2011 als „second awakening“ betitelt,

3 Hier ist „Arab Awakening“ zwar der Titel des Sammelbands, im gesamten Buch ist jedoch stets vom „Arab Spring“ die Rede. 
sieht das erste Erwachen erst mit den Revolutionen der 1950er Jahre gegeben, in denen ein revolutionärer Nationalismus zahlreiche Monarchien stürzte, jedoch nur neue autoritäre Regime hervorbrachte. In jedem Fall verweist der Begriff des Erwachens auf regionale Entwicklungen zu einem Streben nach einer guten politischen Ordnung in Form von Reform oder Revolution, die sich für diachrone Vergleiche eignen (beispielsweise Ouaissa 2011).

Ein viel diskutierter Begriff ist der der Revolution, der im arabischen Diskurs als „Arabische Revolutionen“ statt des „Arabischen Frühlings“ der zentrale Sammelbegriff ist und dabei auf alle arabischen Länder angewendet wird, also auch auf diejenigen, in denen es (bisher) nicht zum Sturz des Herrschaftsträgers gekommen ist. Der westliche Diskurs dagegen beschäftigt sich damit, $o b$ es sich um echte Revolution gehandelt habe - und dies auch nur für die Fälle, in denen das Staatsoberhaupt gestürzt wurde. In Ländern, in denen die Ordnung sich aufgelöst hat, wie etwa in Syrien und Libyen, spielen gewaltsame Konflikte, fragile Staatlichkeit, die Rolle externer Akteure sowie die Diskussion um die Zukunft der Schutzverantwortung (responsibility to protect) nach der NATO-Intervention in Libyen die zentrale Rolle (beispielsweise Bank u. Mohns 2012; bin Talal u. Schwarz 2013). In Monarchien kam es aus westlicher Perspektive dagegen „nur“ zu politischen und wirtschaftlichen Reformen, um Proteste einzuhegen. Hieraus entwickelte sich die Diskussion, ob es sich dabei um die Entwicklung hin zu konstitutionellen Monarchien (vor allem in Marokko) handle, um Liberalisierungen, die als Teil einer „Rekonfiguration“ autoritärer Herrschaft angesehen werden sollten (Dalmasso 2012), oder um die nachhaltige Öffnung des diskursiven Raums, die weitergehende Forderungen und Wandel mittelfristig bringen könnten (Hoffmann u. König 2013).

Aber kann man nun im Fall Ägypten und Tunesien von Revolution sprechen? Der Begriff ist so einfach nicht zu klären und hängt, wenn man sich an Kosellecks Begriffsgeschichte orientiert (2006, S. 240-251) mit zwei Erfahrungsbereichen zusammen, die es mitzudenken gilt: dem eines gewaltsamen Aufruhrs, der zu einem konstitutionellen Wandel führt, und dem eines langfristigen Strukturwandels. Damit bezieht sich die Einschätzung also bei ersterem auf ein Ereignis, bei letzterem auf einen Prozess. Was den ersten Bereich angeht, betont Christoph Schumann in seiner Bewertung der Ereignisse vor allem die Komponente des Verfassungswandels. Sein Argument ist, dass die Proteste in Tunesien und Ägypten, die bereits vor 2011 Republiken waren, keinen Wandel ihrer Verfassungsordnung zum Ziel gehabt, sondern die tatsächliche Umsetzung der formal gegebenen Rechte eingefordert hätten. Die Versprechen von Demokratie und Rechtsstaatlichkeit, wie sie in den Verfassungen der autoritären Regime gegeben wurden, sollten nun endlich eingelöst werden. Damit handelte es sich für Schumann um Revolten, die gegen das herrschende Regime aufbegehrten, und nicht um Revolutionen, die eine politische Ordnung stürzen wollten (2013, S. 34). Aber wenn der Anspruch der Proteste war, die Realität der politischen Ordnung radikal zu verändern, sollte man dann tatsächlich dem „Arabischen Frühling“ seine revolutionäre Dimension absprechen?

Es stellen sich zudem die Fragen, wie weit der Verfassungswandel gehen muss, um revolutionär zu sein, und von welchem Zeitrahmen man auszugehen hat. Denn, so argumentiert Lang (2013), um die neue politische Ordnung werde seit 
2011 gekämpft und der Verfassungsprozess in beiden Ländern sei ein fortschreitender politischer Aushandlungsprozess. Diesen präge weiterhin die Besonderheit, auf die Bayat hinweist, dass die Revolutionäre nach dem Sturz des Diktators nicht die Institutionen des alten Systems abgeschafft oder übernommen hätten. Stattdessen hätten sie versucht, die vielen intakt gelassenen Säulen des Regimes (Militär, staatlich gelenkte Medien, Justiz, Wirtschaftseliten) zu einer selbstständigen Reform zu zwingen, wofür Bayat den Begriff „refolutions“ als Mischung aus „Reform“ und „Revolution“ in die Debatte einführt (Bayat 2013, S. 594-597). ${ }^{4}$

Die Revolution als Ereignis mit einem bestimmten Ergebnis zu sehen wird jedoch immer wieder kritisiert, so zum Beispiel bei Berman (2013). Die Proteste von 2011 als Anfangspunkt einer linearen Demokratisierung zu verstehen, die direkt ohne Umwege und ohne autoritäre „Erblast“ quasi aus einer tabula rasa Situation gedacht wird, sei völlig ahistorisch. Dagegen zeichnet sie an europäischen Beispielen wie der Französischen Revolution nach, welche Umwege postrevolutionäre Verläufe schon immer genommen hätten (siehe auch Shihade 2012, S. 60). Dabei zeige sich die enttäuschte Erwartung auf eine schnelle, direkte Stabilisierung eines demokratischen Regimes als ebenso wiederkehrendes Phänomen. Dies bringe konservative Kräfte dazu, mit den ersten Rückschlägen die Demokratieunfähigkeit des jeweiligen Volkes an sich heraufzubeschwören und autoritäre Zeiten zurückzuwünschen; ein Phänomen, das sich in der Tat auch bei der Bewertung der aktuellen Prozesse in der arabischen Welt findet. ${ }^{5}$

Den Grad der politischen Umwälzung als Maßstab für die Verwendung des Revolutionsbegriffs zu nehmen ist also höchst problematisch. Dies scheint jedoch im etymologischen Ursprung des spätlateinischen revolvere verhaftet zu sein, also „umdrehen“, „zurückwälzen“. Der arabische Begriff thaura vom Verb thara meint in der Grundbedeutung „aufgewirbelt sein “, „aufgeregt sein“, oder im Verbalstamm athara „veranlassen, dass etwas hochfliegt“. Damit passt er sehr gut zu den Ereignissen seit 2011, die die MENA-Region zweifellos aufgewirbelt haben. Begriffsgeschichtlich wird thawra im Sinne von „Revolution“ seit dem letzten Viertel des 19. Jahrhunderts verwendet und hat dabei die positive Konnotation eines Befreiungskampfes (Rebhan 1986, S. 116-117). Diese hat er immer noch inne, wenn politische Akteure reklamieren, die Revolution zu vertreten und zu verteidigen, womit sie ihr politisches Handeln zu legitimieren suchen. Die Revolution zu verraten gilt hierbei als Vorwurf und dient als Grund zum Ausschluss des politischen Gegners vom weiteren politischen Prozess. Hierbei dominiert das Verständnis der andauernden Revolution (al-thawra al-mustamirra) (Harders 2011, S. 33). ${ }^{6}$

4 Dabei versucht Bayat bewusst „refolutions“ von Garton Ashs Verwendung des Begriffs als Reformen eines Regimes, die eine Revolution hervorrufen, zu lösen.

5 Beispielsweise Wallstreet Journal (2013).

6 Die Umwälzung, mit der der Begriff „Revolution“ im westlichen Sprachgebrauch konnotiert ist, findet sich am ehesten im Begriff , inqilab“. Dieser wird jedoch negativ verwendet, zum Beispiel für das Eingreifen der Militärs im Sinne eines Putsches. Wiederkehrend findet sich auch der Terminus „Aufstand“ (uprising), arabisch intifada. Zwar ist der arabische Begriff eng mit dem palästinensischen Widerstand verbunden, kann aber trotzdem als mögliche Vorstufe zu einer „echten“ Revolution - im Sinne des langfristigen Wandels - verstanden werden, sollte sich dieser einstellen (so z. B. Tariq Ramadan 2012). 
Zusammenfassend ist es sicherlich richtig, dass die Suche nach der geeigneten Terminologie zum jetzigen Zeitpunkt nicht abzuschließen ist, da auch die Entwicklung der politischen Verhältnisse im Fluss ist (Beck u. Hüser 2013). Daher ist eine beständige Reflexion der politischen Begriffe nötig, damit sie für unterschiedliche Prozessverläufe offen bleiben und nicht zu vorschnellen Fixierungen führen. Zudem können die Begriffe selbst Gegenstand politischer Auseinandersetzungen werden, wie am Beispiel Ägypten beim Streit darüber sichtbar wurde, ob die Absetzung des Präsidenten Muhammad Mursi nun ein Putsch (inqilab) oder eine Revolution (thawra) gewesen sei.

\section{Die Bedeutung der Proteste von 2011 für das Verständnis von Autokratien}

Mit dem Ausbruch der Massenproteste und dem Sturz einiger Herrscher 2011 kam die öffentliche Kritik an der bisherigen nahostbezogenen Forschung auf, dies nicht vorausgesagt und stattdessen unveränderbare Regimestabilität angenommen zu haben. Diesen Vorwurf konnte die nahostbezogene Forschung mit Verweis auf andere Revolutionen abwehren, die allesamt als Überraschungen für die jeweiligen Beobachter eingetreten waren (Schumann 2013; Bayat 2013, S. 588). Zudem habe man zwar die Dauerhaftigkeit von Regimen erklärt, aber auch auf bestehende Legitimitätskrisen hingewiesen (Schlumberger et al. 2013). Dennoch haben die Proteste von 2011 die Notwendigkeit deutlich gemacht, Dynamiken in Autokratien genauer zu verstehen. So wurden neue Akteursgruppen und Prozesse als untersuchungsrelevante Faktoren einbezogen und das komplexe Zwischenspiel von politics from above und politics from below in den Blick genommen. Aber auch hier ist eine eindeutige Bewertung von Akteuren und Prozessen angesichts der Ergebnisoffenheit der Transformationen schwierig, wie sich an folgenden Debatten zeigt.

Die zentralen Akteure in den Protesten 2011 hatten zuvor in der Forschung kaum Aufmerksamkeit genossen, allen voran Jugendliche, die bis dahin vorwiegend als Alterskohorte und nur als passives Opfer des Bevölkerungsanstiegs und der Jugendarbeitslosigkeit betrachtet worden waren. In den Aufständen von 2011 wurde sichtbar, wie Jugendliche zu Akteuren des Widerstands wurden (Gertel u. Ouaissa 2014). Pauschal von „der Jugend“ zu sprechen, wenn man die Entwicklung der Proteste verstehen will, sei jedoch zu breit und unspezifisch, um den Dissens empirisch zu untersuchen (Allegra et al. 2013, S. 160). ${ }^{7}$ Pace und Cavatorta sprechen von „leaderless horizontal youth-driven networks“ (2013, S. 129). In der Tat zeigen eine Reihe von Arbeiten zu einzelnen Jugendbewegungen, wie ausdifferenziert, historisch gewachsen und kontextabhängig das Phänomen „Jugendbewegung “ im Nahen Osten ist (Gertel u. Ouaissa 2014; Hoffmann u. König 2013; Bouziane u. Lenner 2013). Nationale Belange wie der palästinensische

7 Auch die aktive Teilnahme von Frauen an den Protesten wurde als Überraschung wahrgenommen. Seit 2011 bekam dadurch die Aushandlung der Geschlechterbeziehungen neue Dynamik. Dies kann hier aufgrund des Fokus des vorliegenden Artikels auf die politische Entwicklung und dem weiterbestehenden Gender-Gap in den politischen Transformationen nicht weiter behandelt werden. Es sei jedoch exemplarisch verwiesen auf Al-Ali (2012) und Moghadam (2013). 
Widerstand oder Sezessionsbestrebungen im Südjemen führten ebenso zu Mobilisierung und Organisation von Jugendlichen wie globale Probleme wie die Weltwirtschaftskrise in ihrer lokalen Form von steigenden Preisen, niedrigen Löhnen und Arbeitslosigkeit. Widerstand wurde aber nicht nur in Protesten und politischem Aktivismus artikuliert, sondern auch in den Formen verschiedener Subkulturen wie die des Hip Hop oder Heavy Metal. Dabei sind die stark mit der Jugend assoziierten social media zwar wichtig für die Informationsverbreitung, Vernetzung und Organisation von Protesten, aber erst in ihrer Bedeutung für Akteure und in ihrer Einbettung in Kontexte in ihrer Wirkung zu verstehen (Gertel u. Ouaissa 2014). Die Verengung auf die „Facebook-Jugend“ wurde dabei unter anderem von Shihade kritisiert, weil so nämlich die Proteste nur mit dem assoziiert würden, was westlichen Betrachtern bekannt und gut erscheine, allen voran die Nutzung „westlicher" virtueller Medien und Netzwerke (Shihade 2012, S. 62). Weiterhin nehme man vor allem die Vertreter der Jugend wahr, die Englisch sprechen und zur Teilnahme an einem globalen intellektuellen Diskurs fähig seien (El-Mahdi 2011).

Es zeigt sich also, dass Jugendbewegungen durchaus Akteure des Widerstands sind. Aber im Vergleich zur zentralen Rolle bei den Massenprotesten hat nach der Absetzung der Herrscher "die Jugend" in verschiedenen Formen relativ wenig Einfluss auf die politische Entwicklung genommen. Wie erwähnt, weist Bayat auf die Besonderheit hin, dass die federführenden Jugendbewegungen nach dem Sturz der Herrscher keine politischen Ämter direkt beanspruchten, sondern die Einführung demokratischer Institutionen wie Wahlen und Verfassungen von Akteuren und Institutionen des alten Regimes forderten (Bayat 2013). Hinzuzufügen wäre jedoch die Möglichkeit, dass Jugendbewegungen sich zunehmend politisch professionalisieren und erst Generationen später an politische Spitzenämter gelangen, was weitere Forschung zu Jugendbewegungen langfristig nötig macht.

Im Gegensatz zu den Jugendbewegungen war die aufkommende Arbeiterbewegung bereits vor der Revolution zunehmend in den Blickwinkel der Forschung gerückt (Hopkins 2009; El-Mahdi 2010; Beinin u. Vairel 2013), bevor sie als relevanter Akteur hinter den Aufständen sichtbar wurde (Pace u. Cavatorta 2013, S. 129). So waren in Ägypten politisierte Arbeiter aus unabhängigen Gewerkschaften und einzelnen Arbeiterstädten bei der Organisation der ersten Tage der Proteste in Kairo zwar beteiligt, ihre besondere Bedeutung zeigte sich aber erst mit einem dezentralen Massenstreik, der den Druck auf den Präsidenten Husni Mubarak kurz vor seinem Rücktritt erheblich steigen ließ. Zudem sahen sich die ägyptischen Arbeiter durch die zunehmende Zahl an Streiks und Sit-ins seit 2004 vor allem als Wegbereiter der Revolution (Weipert-Fenner 2013). Im Gegensatz dazu waren in Tunesien lokale und regionale Einheiten des Gewerkschaftsverbands in marginalisierten ruralen Gebieten schon hinter den ersten Protesten nach der Selbstverbrennung Muhammad Bouazizis eine treibende Kraft (Preysing 2013). In Syrien wiederum, so zeigen Leenders und Heydemann (2012), entstanden die ersten Proteste entlang von Clanstrukturen und richteten sich gegen die Brutalität lokaler Regimevertreter. Diese weiteten sich unter anderem durch syrische Arbeitsmigranten in Jordanien und im Libanon aus, die Informationen und Ressourcen für weitere Proteste über die Grenze brachten. Auch diese Beispiele 
zeigen, wie kontextabhängig die jeweilige Rolle von Arbeitern in den Ausbrüchen der Protestbewegungen 2011 war. So warnen Beinin und Vairel zu Recht davor, soziale Bewegungen wie die der Arbeiter automatisch mit Demokratisierung in Verbindung zu bringen (2013, S. 15). Dies gilt auch für sogenannte non-movements, kollektives Handeln in diffuser, aber simultaner Form, wie spontane Proteste gegen Preisanstiege bei Grundnahrungsmitteln, die laut Bayat zwar Widerstand erzeugten $(2010 ; 2013)$, deren politische Auswirkung m. E. jedoch nicht vorauszusagen ist.

Die neue Aufmerksamkeit für gesellschaftliche Akteure in autoritären Regimen führte auch wiederholt zu Kritik an der bisherigen Konzentration auf Eliten in der Autoritarismusforschung. Vorsicht ist jedoch geboten, wenn die Untersuchung der Herrschaftselite und formaler politischer Institutionen als solche verworfen wird, denn Entwicklungen innerhalb der Herrschaftselite können dazu beitragen, sowohl den Umsturzmoment als auch die unmittelbaren Resultate zu erklären. Dies ist umso wichtiger, als es, wie oben dargelegt, nirgendwo zu einem kompletten Austausch der Eliten kam und viele Institutionen des alten Regimes fortbestehen. So können beispielsweise Konflikte zwischen verschiedenen Elitengruppen in Ägypten vor der Revolution erklären, warum das Militär 2011 Teile der regimenahen Wirtschaftselite aus dem politischen System entfernte und damit von einer verbreiteten Ablehnung der Wirtschaftsoligarchen innerhalb der Regierungspartei profitierte (Demmelhuber 2013; Weipert-Fenner 2014). Weiterhin wirken alte Herrschaftspraktiken auch nach dem Sturz der Diktatoren fort wie die des divide et impera. So zeigt Daniel Brumberg, dass bewusst geschürte Identitätskonflikte aus den autoritären Regimen in den Transformationsprozessen fortgesetzt werden. Autokratische Eliten konstruierten und instrumentalisierten jene auf verschiedene Weise, um ihre Herrschaft zu sichern. Dabei kann dieser Konflikt der zwischen religiösen und säkularen Kräften sein, der zwischen verschiedenen Religionen, wie etwa Christen gegen Muslime, oder auch der innerhalb des Islams zwischen Schiiten und Sunniten. Nach dem Sturz des Diktators führen diese Identitätskonflikte die Transformationsprozesse oft in Sackgassen und verschieben politische Inhalte in den Hintergrund (Brumberg 2013).

Die Frage ist also nicht, ob man Eliten und formale Institutionen untersuchen sollte, sondern wie deren Analyse in ein plausibleres Verständnis von Prozessen eingebettet werden könnte. So bemängeln Pace und Cavatorta die bisher dominante top-down Perspektive auf die Regime, in der Institutionen und Politiken stets nur von oben betrachtet und aus der Logik der Eliten heraus im Sinne der Herrschaftsstabilisierung interpretiert wurden. Wahlen, Parlamente oder Verfassungen wurden mit der Intention der Eliten erklärt, das Regime als Ganzes zu stabilisieren, und dieses Motiv mit der tatsächlichen Wirkung der Institutionen gleichgesetzt. Damit wurden die Reaktionen gesellschaftlicher Kräfte sowie nicht intendierte Konsequenzen ausgeblendet (Pace u. Cavatorta 2013, S. 127). Diese Kritik kann man auf politische Gruppen innerhalb der Regierungspartei und der Oppositionsparteien ausweiten, deren Handlungsmöglichkeiten ebenso zu wenig auf ihre Konfliktträchtigkeit untersucht wurden. Wenn man jedoch den Kreis der Akteure mit Handlungs- und Veränderungspotential vergrößert und den Fokus auf die Interaktionen als potentiell konfliktträchtige Auseinandersetzungen legt, 
ist die Frage, wie und, damit verbunden, wo man diese Prozesse beobachten kann. Komplexe Interaktionen und deren Veränderungen zu entdecken und zu bewerten - so unter anderem Allegra et al. - macht zunächst einmal längere Untersuchungszeiträume nötig (Allegra et al. 2013). Zweitens sind die kontextabhängigen $\mathrm{Zu}$ gänge zur Beobachtung dieser Prozesse zu finden. Ein generelles Plädoyer, stärker auf lokale Politiken gerade in der Peripherie autoritärer Regime zu sehen, findet sich gestützt durch zahlreiche Fallstudien bei Hoffmann, Bouziane und Harders (2013). Aber auch speziell Städte können als Zugang zu einem „pool of institutional, informational, relational, and material resources " gesehen werden, der von verschiedenen Akteuren genutzt werden könne, um Dissens zu artikulieren (Allegra et al. 2013, S. 1681). Weiterhin ist das Parlament ein Ort, der Aushandlungsprozesse innerhalb der Herrschaftselite, aber auch mit Oppositionsparteien, sichtbar machen kann (Weipert-Fenner 2014).

All diese Ansätze betonen die „dynamic, ambivalent, and open-ended processes of transformation" (Hoffmann et al. 2013, S. 2). Diese werden noch einmal verdeutlicht, wenn man die gängige Erwartung auf einen Regimewandel vor 2011 betrachtet. Weit verbreitet war die Annahme, dass wirtschaftliche Liberalisierung, wie sie in den meisten Ländern der MENA-Region in den 2000er Jahren verstärkt unternommen wurde, zu Modernisierung und Fortschritt führen und dabei eine pro-demokratische Mittelklasse hervorbringen würde. Als der Wandel ausblieb, sank die Erwartung auf Reformen, die vom Westen gefordert wurden und von reformwilligen Regimeeliten umgesetzt werden würden (Bayat 2013). Diese Überbleibsel der Modernisierungstheorie überdeckten, dass die tatsächlich durchgeführten Wirtschaftsreformen zur Entstehung einer regimenahen Oligarchie und zu einer immer größer werdenden sozialen Ungleichheit führten, deren Ausdruck die erwähnten sozialen Proteste waren (Zorob 2013). Damit hatten die Wirtschaftsreformen indirekt durchaus auf einen Demokratisierungsprozess hingewirkt, aber offensichtlich nicht auf lineare Weise (Pace u. Cavatorta 2013, S. 129-130). Diese Beobachtung lässt sich verallgemeinern und als ein Plädoyer für ein Verständnis von Prozessen verstehen, das den nicht-linearen, langfristigen Wandel in den Blick nimmt und Prozesse nicht als Kausalkette von Strukturmerkmalen organisierter Akteure und Strukturen ableitet (Harders 2011, S. 11). Für die weitere Forschung bedeutet dies politics from above - und die dazugehörigen weiterhin aussagekräftigen Ansätze zum Rentierstaat und dem Neopatrimonialismus (Schlumberger et al. 2013) - mit der neuen Aufmerksamkeit für politics from below zusammenzuführen (Asseburg et al. 2012) und empirisch sehr genau die Wechselwirkung von Akteuren, Strukturen und Diskursen zu analysieren (Harders 2011, S. 15-16).

Die Ergebnisoffenheit von Entwicklungen im autoritären Regime anzunehmen, die sich aus potentiellen Konflikten und deren Eigendynamiken, aus nicht-intendierten Konsequenzen und Kontingenz ergibt, wird im Rückblick vermisst. Sie ist aber auch im Hinblick auf die weiter existierenden Autokratien nicht automatisch gegeben. Ein Beispiel dafür ist die aktuelle Debatte, warum nur Herrschaftsträger in Republiken, aber nicht in Monarchien gestürzt wurden. Schnell wird hierbei die Annahme dauerhafter Regimestabilität nicht mehr von Autokratien insgesamt, sondern nun von einem bestimmten Regimesubtyp, abgeleitet. Aus Huntington's king's dilemma (1968), dass Monarchien nicht modern und damit per se instabil 
seien, wird der king's advantage konstruiert, der erkläre, dass gerade die NichtölMonarchien in Jordanien und Marokko ein höheres Maß an Legitimität durch einen auf Religion und Tradition begründeten Herrschaftsanspruch hätten als Präsidenten und damit generell vor Revolutionen gefeit seien. ${ }^{8}$ Differenziertere Analysen zur Stabilität von Monarchien wie von Bank und Richter (2013) beziehen gescheiterte Königtümer im Nahen Osten zwischen 1945 und 2011 mit ein und erkennen hier einen Zusammenhang zwischen der Stabilität von Monarchien und bestimmten Kombinationen von internen und externen Faktoren. So hätten sich für die Golfmonarchien Renteneinnahmen und familiäre Beteiligung an der Herrschaft auf der einen Seite und für Jordanien und Marokko externe Unterstützung und auf Religion und Staatsgründung basierende Legitimität auf der anderen Seite als stabilitätsfördernd erwiesen. Doch auch hier stellt sich die Frage, wo Raum für Entwicklungen in den Monarchien ist. Davidson (2012) geht von einem nahenden Kollaps der Golfmonarchien aufgrund der Dynamiken, die der Rentierstaat verursache, aus. Bei pro Kopf schrumpfenden Ausgaben sei der Tausch ökonomischer Privilegien gegen politische Partizipation nicht ewig aufrechtzuerhalten. Außerdem seien die Renteneinnahmen auch in Repressionsapparate geflossen, deren Aktivierung beim Aufkommen von Protest zunehmend auf besser vernetzte und informierte Bürger träfe, die Einschüchterung nicht von weiterer Kritik abhalte. Auch in Marokko (König u. Hoffmann 2013) und Jordanien (Bouziane u. Lenner 2013) würden die Grenzen für öffentliche Regimekritik immer weiter ausgedehnt und die daraus resultierenden Dynamiken könnten nicht abgesehen werden.

In den eben skizzierten Verengungen der Autoritarismusforschung sah eine postkoloniale Kritik alte Stereotypen über die MENA-Region und in den Protesten von 2011 die Chance, diese sogenannten Orientalismen zu überwinden. Zunächst plädiert unter anderem Teti (2012a) für die Überwindung des „Middle East exceptionalism “ in der Demokratisierungsliteratur, in der die Region, die den vorangegangenen Wellen der Demokratisierung widerstanden hatte, als per se demokratieunfähig dargestellt worden sei. Einen der Gründe fand man, verstärkt durch den 11. September 2001, im angeblich antidemokratischen Islam. Dabei sei ein universalistisches Modell von Demokratie angenommen worden, das ein bestimmtes Bild von Säkularismus beinhalte, das kein vom - vermeintlich einheitlichen - westlichen Modell abweichendes Verhältnis von Religion und Politik zulasse (Teti 2012a; Stepan u. Linz 2013). Diese essentialistische Sichtweise auf eine Religion und Region knüpfte an alte Stereotype vom „Orient“ als mittelalterlich, starr und unreif an, die die Erwartungen, wie es mit der MENA-Region weitergehen würde, beeinflussten. Diese Stereotype jedoch, so verkündeten Vertreter einer postkolonialen Perspektive, seien nun endgültig vom Tisch, eine neue Sprache und Geografie würden sich etablieren, die von Orientalismen befreit seien (Dabashi 2012). Die ersten warnenden Stimmen kamen doch schnell auf, die auf neo-orientalistische Konstruktionen verwiesen. Besonders die Fokussierung auf "die Jugend" als Hauptträger der Revolutionen wurde, wie bereits erwähnt, dafür kritisiert, dass sie diese gleichsetzte mit der englischsprachigen Mittel- und Ober-

8 Ein Überblick über die Debatte findet sich beispielsweise bei Yom (2012). 
schicht, die einer „westlichen“ Jugend samt Mediennutzung ähnelte (Shihade 2012, S. 62; El-Mahdi 2011). Die noch deutlichere Rückkehr der Stereotype scheint jedoch in dem Moment stattzufinden, in dem sich große Ernüchterung über die Demokratisierungsprozesse breitmacht und dazu verleitet, dies mit der prinzipiellen Unfähigkeit der MENA-Region zur Demokratie zu erklären. Gegen die bekannten Orientalismen, wenn auch in neuem Gewand, könnten interregional vergleichende Studien helfen, zu hohe Erwartungen wie den unmittelbaren Eintritt von Demokratie gegen die Offenheit für unterschiedliche, auch nichtlineare Verläufe von Demokratisierungsprozessen einzutauschen, wie sie beispielsweise Osteuropa oder Lateinamerika erfahren haben.

\section{Demokratieförderer oder -verhinderer? Die ambivalente Rolle interner und externer Akteure für die Transformationen in Ägypten und Tunesien}

Wie schwierig es ist die aktuellen Entwicklungen in große Zusammenhänge wie Demokratisierung oder Rückkehr zum autoritären Regime einzuordnen, zeigt sich noch einmal deutlich in den Analysen der Fälle, in denen das Regimeoberhaupt gestürzt wurde. Am Beispiel von Tunesien und Ägypten soll im Folgenden dargelegt werden, wie ambivalent die Rolle bestimmter Akteure ist, die für die Demokratisierung sowohl förderlich als auch hindernd sein können. Dies mag auch dazu beitragen, die Nicht-Linearität der Transformationen in Bezug auf Demokratie zu unterstreichen und den bisherigen Verlauf, der auf den ersten Blick als „ein Schritt vor und zwei zurück“ erscheinen mag, plausibler zu machen.

\subsection{Die Wiederentdeckung der Armee}

$\mathrm{Zu}$ den am meisten diskutierten Themen der Transformation zählt die Rolle des Militärs, da es die unterschiedlichen Entwicklungen vor allem im Hinblick auf die Dauer der Proteste, die politische Intensität und das Ausmaß an Gewalt stark beeinflusste (Frisch 2013, S. 177-179). Hatte nach dem Zweiten Weltkrieg bis in die 1970er Jahre hinein der „prätorianische Staat" samt zahlreichen Militärputschen die MENA-Region geprägt, so war es in den darauf folgenden Jahren zu einem Rückzug der Armee von der politischen Bühne gekommen. Dies war jedoch unter sehr verschiedenen Parametern erfolgt und reichte von gezielter politischer und wirtschaftlicher Marginalisierung der Streitkräfte bis zur Einhegung gegen Kompensation. In allen Fällen war die Armee im politischen Tagesgeschäft direkt nicht mehr sichtbar, was auch das Interesse der Forschung zurückgehen ließ. Dies hat sich nun geändert. Aurel Croissant beispielsweise erklärt überblickshaft anhand unterschiedlicher Ansätze der Putschprävention durch autoritäre Herrschaftseliten und den daraus folgenden militärisch-politischen Beziehungen die drei beobachteten Phänomene seit 2011: Spaltung der Armee (Libyen, Jemen), Loyalität der Armee zum Regime (Syrien, Bahrain), Ausstieg der Armee aus der Regimekoalition (Tunesien, Ägypten) (2013, S. 105). Letzteres Phänomen hat besondere Aufmerksamkeit erhalten, da Armeen im Bündnis mit prodemokratischen Aktivisten zunächst überraschten. Was in Tunesien mit der von Ben Ali politisch wie wirtschaftlich marginalisierten Armee noch verständlich wirkte, bedurfte im Fall 
Ägyptens, wo die Armee ein relativ saturierter Bestandteil der Herrschaftselite war, genauer Betrachtung. Albrecht und Bishara zeichnen den Übergang von der Armee als Krisenmanager der inneren Sicherheit in den ersten Tagen der Proteste über die Ausübung des politischen Managements hin zu dessen Institutionalisierung nach (2011). Die veränderte Wahrnehmung der Lage seitens der Generäle durch die Zunahme der Proteste und damit eine Neuausrichtung ihres politischen Verhaltens zur Wahrung der armeeeigenen Interessen werden verständlich, wenn sie in den Kontext des sogenannten deep-state eingebettet werden. Damit ist eine enge Verflechtung zwischen zivilen und militärischen Sektoren gemeint, deren Entstehung in Ägypten bei Albrecht (2013) erläutert wird. Diese Verflechtung mache die wiederkehrende Intervention der Armee in die Politik wahrscheinlich, wenn die „Komfortzone“ der Armee samt politischer Mitsprache und wirtschaftlicher und institutioneller Autonomie bedroht sei. Ob das Interesse gegeben sei, dauerhaft in der ersten Reihe zu stehen, erscheine aus der langfristigen Entwicklung der Armee heraus „vom Herrschaftsträger zur Herrschaftsstütze“ (Croissant 2013, S. 99) unwahrscheinlich. Allerdings stellt sich die Frage, ob nicht doch persönliche Ambitionen auf politische Führungspositionen bei Generälen überwiegen könnten, wie es beim Beispiel des aktuellen Personenkults um den Oberbefehlshaber der ägyptischen Streitkräfte Abd al-Fattah al-Sisi nahezuliegen scheint.

Eine zentrale Variable in den Beziehungen des zivilen und militärischen Sektors ist die Verzahnung mit der Wirtschaft. Springborg (2011) stellt für die Armeen des Nahen Ostens ein Spektrum auf, an dessen äußersten Ende Ägypten und - oft vernachlässigt - der Iran mit sogenannten „Military, Inc.'s“ stehen. Diese sind gekennzeichnet durch parallele Offiziersökonomien. Er stellt weiterhin eine negative Korrelation zwischen militärischer Verbindung mit der Wirtschaft und der Professionalität der Truppen sowie deren Kontrolle durch zivile Institutionen auf. Damit zeigt sich die ambivalente Rolle der Armee für den Demokratisierungsprozess: Ohne ihr Ausscheren aus der Herrschaftskoalition kommen Diktatoren ohne systematische Gewalt nicht zu Fall. Gleichzeitig erschwert das Gewicht der Armee die Möglichkeit, sie unter demokratische Kontrolle zu bekommen.

\subsection{Politischer Islam: neue Positionen, neue Akteure}

Für die Forschung zum politischen Islam bot sich durch die Umbrüche in Tunesien und Ägypten zunächst die Gelegenheit, die seit Jahren geführte Debatte über die Demokratiefähigkeit von Islamisten mit der empirischen Beobachtung von Islamisten an der Regierungsmacht anzureichern. Waren die schönen Worte zu Menschenrechten und Demokratie, die sowohl die tunesische Nahda als auch die ägyptischen Muslimbrüder unter der Diktatur vertreten hatten, mehr als ein strategisches Kalkül, um liberale Ängste zu lindern und, erst einmal an der Macht, doch einen islamischen Gottesstaat auszurufen? Bis heute dominiert die Auseinandersetzung zwischen Islamisten und säkularen beziehungsweise „zivilen“ ( $m a$ dani) Kräften, wie es im ägyptischen Diskurs heißt, den Transformationsprozess. Die Polarisierung hat in Tunesien und Ägypten kontinuierlich zugenommen und erweckt schnell den Eindruck, dass sich hier zwei unvereinbare politische Ordnungsvorstellungen gegenüberstünden. Das Narrativ des „die oder wir“, das be- 
reits die autoritären Vorgänger geschickt einzusetzen wussten, um dem Demokratisierungsdruck mit Verweis auf die Islamisten als einzige Alternative zu entgehen, kommt nun in neuem Gewand daher und stellt Vertreter des politischen Islam als nicht demokratiefähig dar.

Gegen diese Pauschalisierung sprechen jedoch zahlreiche Analysen. Zum einen führte die neue Freiheit nach dem Sturz der Diktatoren als direktes Ergebnis nicht zu einem gemeinsamen Block von Islamisten, sondern vielmehr zu einer Pluralisierung des Akteursspektrums. Die bis dahin politisch stärkste Organisation, die Muslimbruderschaft, gründete eine separate Partei, ihre Jugendbewegung sowie einzelne Vertreter der Muslimbruderschaft trennten sich von ihr. Eine frühere Abspaltung der Bruderschaft, die Wasat Partei, wurde offiziell zugelassen (Lübben 2013). Ehemals militante Gruppen gründeten Parteien, ebenso wie die bis dahin quietistischen Sufis, die sich aber bisher in Wahlen kaum durchsetzen konnten (Wirth 2013). Und drittens gibt es ein breites Feld an salafistischen Parteien, deren Mitglieder sich bis zur Revolution allein auf missionarische und soziale Aktivitäten konzentriert hatten (Brown 2011). Neben der Ausdifferenzierung der Akteure sind die Islamisten auch nicht ideologisch starr im Denken und Handeln, sondern stellen sich als anpassungsfähige, pragmatische und rationale Akteure dar. Dalmasso und Cavatorta zeigen dies im Vergleich des Verhaltens der Nahda und der islamistischen PJD (Parti de la justice et du développement) in Marokko bei den jeweiligen Verfassungsprozessen (2013). Auch die ägyptische Muslimbruderschaft wurde zeitweise für ihren Pragmatismus gelobt, sei es bei der vermittelnden Rolle zwischen Hamas und Israel im Oktober 2012 oder bei dem Arrangement, das sie mit der Armee im August 2012 schloss, um die Generäle aus der ersten Reihe der Politik zu entfernen. Auch bei den Salafisten lassen sich Pluralisierung und Pragmatismus erkennen. Al-Anani und Malik geben einen Überblick über die verschiedenen Gruppen sowie deren Entstehung und Verhältnis zueinander (2013). Die Anpassungsfähigkeit an neue Bedingungen wird beispielsweise darin sichtbar, dass vor der Revolution Demokratie als unislamisch abgelehnt wurde, nach der Revolution aber nun umgedeutet wird zum Instrument, Ägypten islamischer zu machen. Partizipation wird sogar zur Notwendigkeit (darura) erklärt. Damit sehen die erwähnten Autoren, dass die Öffnung der politischen Arena zu einer Zähmung radikaler Teile der Salafiya geführt habe, die sich sogar explizit vom saudischen Modell der Salafiya abgrenzten (2013, S. 67). Diese zunächst positiv anmutenden Einschätzungen stehen jedoch im Kontrast mit dem zunehmend düsteren Bild von der Muslimbruderschaft, das sich in der öffentlichen Wahrnehmung der Muslimbruderschaft entwickelt hat. Mit dem Sturz Muhammad Mursis sind die Muslimbrüder und ihre Partei wieder aus dem politischen Prozess ausgeschlossen und die weitere Entwicklung der Bruderschaft und des gesamten islamistischen Spektrums erscheint wieder völlig offen. Auch in Tunesien hat sich ein negatives Bild von der Nahda-Bewegung und deren Partei herausgebildet und die starke Polarisierung zwischen Islamisten und säkularen Akteuren, insbesondere in Anbetracht der zunehmenden politischen Gewalt, bedroht den politischen Wiederaufbau.

Ist diese Ernüchterung nun doch der Beweis für die Demokratieunfähigkeit der Islamisten? Dagegen argumentiert Malika Zeghal, die für Tunesien das nachzeich- 
net, was Brumberg (2012) als Identitätskonfliktlinien des alten Regimes bezeichnet: Im Zuge einer spiegelbildlichen wechselseitigen Stereotypisierung würden die Säkularen den Islamisten unterstellen, allein die Unterschicht anzuziehen und eine rein arabische und muslimische Identität zu konstruieren. Die Islamisten wiederum sähen die Säkularen charakterisiert durch Frankophonie sowie einer Zusammensetzung aus postkolonialen und intellektuellen Eliten und Alliierten des alten autoritären Regimes, die blind das westliche Leben imitieren würden. Mittlerweile habe sich der Konflikt konzentriert auf die Verteidigung der Meinungsfreiheit durch die Säkularen gegen ein von den Islamisten definiertes Verständnis öffentlicher Ordnung, das als nicht verhandelbar erscheine und teilweise in Gewalt ende (Zeghal 2013, S. 267-270). Das Interessante an Zeghals Analyse ist, dass sie parallel dazu die soziologische Analyse der Wählerschaft beider Lager heranzieht, die diesen Stereotypen widerspricht und zeigt, dass beide, die Nahda und säkulare Mitte-links-Parteien von urbanen und gebildeten Gruppen gewählt wurden, ärmere Schichten dagegen populistischen Listen ihre Stimme gaben. Die Überwindung dieser an einen Kulturkampf erinnernden Konstellationen wird auch in Ägypten eine Herausforderung sein, an der auf längere Sicht kein Weg vorbeiführt. Wie Donker (2013) darlegt, handelt es sich hier um eine Reihe von Fragen zur Rolle der Religion für Staat und öffentliche Ordnung, die als Aushandlungsprozess nicht nur zwischen Säkularen und Islamisten, sondern auch zwischen den verschiedenen Akteuren im politischen wie auch sozialen Spektrum des islamischen Aktivismus zu verstehen sind. Diese Pluralität verdeutlicht einmal mehr die zwangsweise Offenheit der Entwicklung und warnt vor einer normativen Einstellung bezüglich der Rolle bestimmter Akteure für den Demokratisierungsprozess. Dies fällt jedoch oft nicht leicht, unter anderem aufgrund der stark polarisierten Medienlandschaft als ein wichtiger Zugang zu den Prozessen.

\subsection{Die Rolle der Medien}

Wie die Medien zur Entstehung der Proteste 2011 beitrugen, ist ein zentraler Punkt, an dem sich eine große Debatte entsponnen hat. Im öffentlichen Diskurs kursierten schnell Begriffe wie „Facebook-“ oder „Twitter-Revolution“ und suggerierten, dass neue soziale Medien die Diktatoren zu Fall gebracht hätten. Damit wird angenommen, dass im Internet die Revolutionen begonnen hätten und das Internet per se diktaturfeindlich und demokratiefreundlich sei. Dagegen weisen die meisten Analysen auf ein sehr viel differenzierteres Bild hin, das verschiedene Medienarten beinhaltet, die auf unterschiedliche und auch ambivalente Weise benutzt werden und auch wechselseitig wirken. Hier geht es darum auszuloten, inwieweit bestimmte Medienarten das Vermögen zu Regimekritik und -wandel gesteigert haben, aber auch wie sie Möglichkeiten für autoritäre Herrschaft geschaffen haben, Zwang und Repression auszuüben. Darüber hinaus können Medien auch jenseits der Regimefrage Wirkung auf zwischen- und innerstaatliche Konflikte haben, die es bei der Bewertung der Rolle der Medien zu bedenken gilt (Brynen et al. 2012, S. 234).

Sieht man sich die digitalen Medien an, so ist die Auseinandersetzung mit ihrer Rolle doch zunächst von einer Sammlung von Funktionen geprägt, die sich auf 
Mobilisierung, Organisation und Austausch von Informationen beziehen. Howard und Hussain (2013, S. 26) teilen diese Funktionen in fünf Phasen ein, die das $\mathrm{Zu}$ sammenspiel von Protesten und digitalen Medien genommen habe: die Vorbereitung, d. h. die Verbreitung des Wissens über die Korruptheit und Brutalität des Regimes sowie alternativer Gegendiskurse, die Entzündung der Proteste durch die schnelle, zensurlose Weiterleitung von Daten wie bestimmter Bilder oder Videos, die Koordination der Straßenproteste, das Einbeziehen internationaler Adressaten und schließlich das Austragen ideologischer Konflikte nach dem Punkt der Entscheidung (Sturz des Diktators, gewaltsame Niederschlagung des Protests, Pattsituation) zwischen Gewinnern und Verlierern.

Diese Funktionen können jedoch mit Sicherheit nicht allein den digitalen Medien zugeschrieben werden. So ist beispielsweise die Rolle von Mobiltelefonen zentral geworden, als die autoritären Regime versuchten, die Kommunikationskanäle der Proteste durch das Abschalten des Internets zum Erliegen zu bringen (Howard u. Hussain 2013, S. 116). Handys stellten generell eine Säule der Informationsgenerierung und -verbreitung durch ihre Foto- und Videofunktionen dar. Ins Internet eingespeist dienten die so generierten Informationen auch analogen Medien als Quellen. Hier entwickelten sich neue Verbindungen zwischen Medienarten, als der regionale Nachrichtensender al-Jazeera beispielsweise Videos und Nachrichten der Protestierenden aus Tunesien und Ägypten übermittelte und damit sowohl unter den Aktivisten als auch in Richtung der internationalen Öffentlichkeit als Kommunikationskanal diente (Brynen et al. 2012, S. 234). Die unermüdliche Berichterstattung über einige Revolutionen durch den Satellitensender bestärkte die bestehenden Annahmen zum „al-Jazeera-Effekt“ darüber, dass Medien die Politik verändern können (bereits Seib 2008, Miles 2011).

Nun könnte dies in eine generelle Euphorie bezüglich des Demokratisierungspotentials der Medien, analog wie digital, münden, aber Brynen et al. weisen auch auf negative Facetten hin. So hätten nicht nur autoritäre Regime die digitalen Medien und besonders virtuelle Netzwerke genutzt, um Aktivisten auszuspähen oder in Fallen zu locken, sondern virtuelle Medien würden dazu verleiten, sich nur noch unter Gleichgesinnten in den eigenen Netzwerken auszutauschen und zu informieren. Dies könne zu Spaltung und Polarisierung beitragen, was einem generellen Trend der arabischen Medienlandschaft entspräche. Rundfunk, Print und Fernsehkanäle, national wie regional, seien unmittelbar nach dem Sturz der Diktatoren als Sprachrohre bestimmter Gruppen sichtbar geworden. Ob es sich nun um den Stellvertreterkonflikt zwischen Qatar (über al-Jazeera) und Saudi-Arabien (al-Arabiyya) handle, oder zwischen Religionen oder politischen Gruppierungen - die Standards der Berichterstattung bezüglich Objektivität und Faktenbezogenheit litten meist gewaltig (Brynen et al., S. 240-245). Dies hänge beispielsweise in Ägypten, so Richter, mit der Medienlandschaft nach den Privatisierungen der 1990er Jahre zusammen, die maßgeblich der Logik gefolgt waren, einerseits die zumindest rhetorisch auf liberale Demokratie pochenden westlichen Staaten zu beruhigen und andererseits die Interessen von Wirtschaftseliten zu befriedigen und sie damit ans Regime zu binden. Somit könnten bis heute auch die meisten privaten Medien nicht als unabhängig betrachtet werden, da sowohl kommerzielle Interessen als auch anti-islamistische Tendenzen der Wirtschaftsoligarchen die 
Ausgewogenheit der Berichterstattung beeinträchtigten. Dennoch hätten die Privatisierungen einen wenn auch begrenzten Freiraum geschaffen, in dem sich vereinzelt unabhängige Medien herausgebildet hätten (Richter 2013). Diese ermöglichten zusammen mit den erwähnten Möglichkeiten durch das Internet das Erschließen neuer diskursiver Räume und indirekte Lernprozesse bei Protestakteuren (Weipert-Fenner 2013).

Damit findet sich in den meisten Beiträgen die Einsicht, dass verschiedene Medienarten für die Transformationsprozesse bedeutsam seien, deren jeweilige Rollen indes positiv wie negativ auf die politische Entwicklung wirken könnten, und dass nicht intendierte Folgen der Mediennutzung zu berücksichtigen seien.

\subsection{Externe Demokratieförderung durch die EU: Lektionen und Reaktionen}

Im letzten Jahrzehnt wurde viel diskutiert über die Möglichkeit, Demokratie von außen in die MENA-Region zu bringen. Doch wie Kodmani (2010) noch vor 2011 zusammenfasste, scheiterten die Versuche der USA und der Europäischen Union (EU) nicht zuletzt an den Problemen mit der eigenen Glaubwürdigkeit. Im Zweifel hatten die Interessen der „Demokratieexporteure“ immer einen höheren Stellenwert als die der Bevölkerungen in den arabischen Ländern, wobei gerade ihre Interessendefinition durch einen kurzfristigen Fokus auf Stabilität in der Region geprägt war. Von multilateralen Verhandlungen mit den autoritären Regimen ging die EU seit Mitte der 2000er Jahre zu einem bilateralen, sektoral differenzierten Ansatz mit positiver Konditionalisierung über. Doch auch hierzu fiel und fällt in den meisten Analysen die Bewertung eher verhalten aus, sah man doch in dem differenzierten Ansatz noch stärker spezifische Interessen der EU (wie Migrationssteuerung und den Transfer des acquis communautaire gerade im Bereich der Marktliberalisierung) vertreten und das Ziel der Demokratie für die südlichen Mittelmeeranrainer hintangestellt (Tömmel 2013, S. 22-23). Neben all der durchaus plausiblen Kritik auch an einer ,arbitrary and largely useless selection of pseudo-benchmarks“ (Del Sarto u. Schumacher 2011, S. 932), die der Demokratieförderung ein objektives und effizientes Image verleihen sollten, ist der Einfluss externer Mächte und ihres wie ernst auch immer gemeinten Werbens für Demokratie nicht völlig bedeutungslos. Wie Kodmani noch vor 2011 erkannte, trug der internationale Druck zu Reformen bei, die in den Autokratien scheinbar ungefährliche Bereiche wie die Liberalisierung der Medienlandschaft betrafen. Der öffentliche Raum für Artikulation und Protest wurde, wie oben ausgeführt, größer und erlaubte damit das Entstehen vieler unterschiedlicher Protestgruppen und -arten, die sich wechselseitig durch indirekte Lernprozesse beeinflussten und verstärkten (Kodmani 2011, S. 158). Gleichzeitig ließ sich beobachten, dass Demokratie als Maßstab in den politischen Diskurs übergegangen war, gleichermaßen auf Seiten der autoritären Herrschaftselite, die sich durch graduelle Reformen in Richtung Demokratie rechtfertigte, wie auf regimekritischer Seite, wo genau dieser Maßstab als beständiges Kriterium zur negativen Evaluation der Legitimität der Herrschaft benutzt wurde. Wie sehr die EU mit dem zunehmenden Fokus auf sektorale Kooperation mit den autoritären Regimen im Namen der Demokratisierung dazu beitrug, ist indes fraglich. 
Bei der recht bescheidenen Bilanz der bisherigen Demokratieförderung stellt sich nun umso drängender die Frage, wie die Demokratieförderer ihre Strategien in dem Moment änderten, als sie offene Türen einrennen konnten. Das Fazit in Bezug auf die EU lautet bisher: so gut wie gar nicht. Laut Tömmel lassen sich Kontinuität und Wandel der Europäischen Nachbarschaftspolitik (ENP) im Zuge der aktuellen Transformationen weniger durch die Veränderungen in den südlichen Mittelmeeranrainern verstehen als durch EU-interne Zwänge. Im Sinne des Neo-Institutionalismus seien diese auch nicht nur zweckrational zu sehen, sondern stünden im Bezug zu dem, was innerhalb der Organisation angemessen sei. Im Fall der EU ergebe sich dies aus einem komplexen Zusammenspiel der Kommission und der Mitgliedsländer, die in Bezug zur MENA-Region unterschiedliche Interessen und Strategien verfolgten (Tömmel 2013, S. 31-34). Ein Lernprozess seitens der EU wird aber zumindest bei der Gestaltung der Umsetzung gesehen. Neben der deutlichen Erhöhung des Finanzrahmens für das Instrument der Europäischen Nachbarschaftspolitik wurden verschiedene Arten von Abkommen, die die ENP bisher vorsah, in einen "Single Support Frame“ zusammengefasst und unter die neuen Slogans „more for more“ und „less for less“ gestellt. Dahinter, so Schumacher, stecke jedoch nicht viel mehr als die Betonung des Ansatzes der positiven und negativen Konditionalisierung der Zusammenarbeit und Hilfsmittel bei Beibehaltung des bilateralen Rahmens. Doch gerade der multilaterale Ansatz, der auch regionale Entwicklung ermöglichen würde und der Mitte der 1990er Jahre noch bei der Entstehung der euromediterranen Partnerschaft zentral war, fehle weiterhin. Dies verhindere ökonomische und infrastrukturelle Integration, die für eine nachhaltige Entwicklung in der Region eine große Chance biete (Schumacher 2012, S. 101102). Wie Teti bemängelt, würde Demokratisierung weiterhin vor allem politisch im Sinne einer liberalen Demokratie mit Fokus auf politische Institutionen und Rechte gedacht. Sozioökonomische Faktoren als einer der Auslöser der Proteste würden zwar genannt, aber eine tiefgehende Analyse der Wirtschaftslage vor der Revolution inklusive einer kritischen Reflexion der neoliberalen Wirtschaftsordnung, die die EU fordert und fördert, finde nicht statt. Somit bliebe auch die Verbindung zwischen Demokratie und sozioökonomischer Gerechtigkeit unberührt, stattdessen werde letztere in den Bereich der Entwicklungspolitik verschoben (Teti 2012b, S. 270). Neben dem Risiko, keinen positiven Einfluss auf die Demokratisierung zu haben, warnt van Hüllen davor, dass weiterhin die Gefahr bestehe, in der Kooperation mit autoritären Regimen diese eher zu stützen als zu schwächen (2012, S. 140). Dieser Vorbehalt, dass die Auswirkungen einzelner Maßnahmen auf die Regimefrage nicht vorherzusehen sind, gilt besonders angesichts der ambivalenten Entwicklungen in den Transformationsländern und passt zu der insgesamt eher negativen Einschätzung bezüglich der Erfolgsaussichten der EU-Demokratieförderung selbst nach dem Sturz von Diktatoren.

\section{Fazit}

Wohin steuert die MENA-Region? Die Massenproteste von 2011 und die diversen Transformationsprozesse haben der politikwissenschaftlichen Nahostforschung einen neuen Schub verpasst, aber eine klare Antwort kann bisher nicht 
gegeben werden. Aus der Ergebnisoffenheit der weiterhin sehr dynamischen Entwicklungen ergeben sich drei Probleme für die politikwissenschaftliche Forschung: Erstens die Suche nach der passenden Bezeichnung: „Arabischer Frühling“, „Arabische Revolution“ - oder doch „nur“ Revolte? Hier finden sich unterschiedliche Annahmen zum Zeitrahmen und dem Ausmaß des politischen Wandels, die nicht zuletzt in den deutschen, angelsächsischen und arabischen Begriffsgeschichten begründet sind. Zweitens führt die Ergebnisoffenheit dazu, dass die Annahmen zum autoritären Regime zwar überdacht wurden, aber eine klare Bewertung von Akteuren und Prozessen weiterhin schwierig bleibt. So haben die Proteste von 2011 die Forschung zu autoritären Regimen zum einen dahingehend angeregt, Akteure jenseits der Herrschaftselite mit dem Potential für Widerstand und Wandel zu sehen, zuvorderst Jugend- und Arbeiterbewegungen. Auch die Annahmen zu politics from above und Zugänge zur Verbindung der Analyseebenen wurden erweitert, sind jedoch konzeptuell noch in der Weiterentwicklung, um Prozesse im autoritären Regime aus der Wechselwirkung von Akteur, Struktur und Diskurs zu erklären. Drittens zeigen die Transformationen in Ägypten und Tunesien, dass Faktoren, die zur Demokratisierung beitragen, ebenso in die andere Richtung zurück zur Autokratie führen können. Dies wird deutlich in Bezug auf die Rolle der Armee, des politischen Islams und der Medien. Auch beim externen Akteur der EU und deren Demokratisierungspolitik wird die ambivalente Wirkung auf die Transformationsprozesse sichtbar.

Dieser Zwischenstand zur ersten „Welle“ an Publikationen zwischen 2011 und 2013 zeigt also die offensichtliche Notwendigkeit genauer empirischer Aufarbeitung sowohl der autoritären Regime unter neuen konzeptionellen Vorannahmen und Zugängen als auch der Akteure und Prozesse in Demokratisierungsprozessen. Es bleibt zu vermuten, dass die nächsten Jahre durch etwas zeitlichen Abstand zum Ausbruch der Proteste im Jahr 2011 und im Zuge der Verdichtung empirischer Beobachtungen mehr konzeptuelle Arbeiten auch anhand interregionaler Vergleiche hervorbringen werden, die über die Regionalforschung hinaus zur allgemeinen Autoritarismus- und Demokratisierungsliteratur beitragen werden.

\section{Literatur}

al-Ali, Nadje. 2012. Gendering the Arab Spring. Middle East Journal of Culture and Communication 5: 26-31.

al-Anani, Khalil, und Maszlee Malik. 2013. Pious Way to Politics: The Rise of Political

Salafism in Post-Mubarak Egypt. Digest of Middle East Studies 22: 57-73.

Albrecht, Holger. 2013. Revolution oder Coup d'État? Die Rolle des Militärs in der ägyptischen Politik. In Revolution und Regimewandel in Ägypten, Hrsg. Holger Albrecht und Thomas Demmelhuber, 63-86. Baden-Baden: Nomos.

Albrecht, Holger, und Dina Bishara. 2011. Back on Horseback: The Military and Political

Transformation in Egypt. Middle East Law and Governance 3: 13-23.

Alexander, Anne, und Mostafa Bassiouny. 2013. Workers and the Arab Revolutions: The Impact of Workers Strikes and Trade Unions in Egypt, Tunisia and Syria. London: Zed Books. 
Allegra, Marco, Irene Bono und Jonathan Rokem. 2013. Rethinking Cities in Contentious Times: The Mobilisation of Urban Dissent in the 'Arab Spring'. Urban Studies 50: 1675-1688.

Asseburg, Muriel, Henner Fürtig, Cilja Harders, Annette Jünemann, Rachid Ouaissa, Friederike Pannewick, Oliver Schlumberger und Christoph Schumann. 2012. Political Science Perspectives and Agendas for Research on the Middle East and North Africa after the Arab Spring. In Realigning Power Geometries in the Arab World. Conference Reader, Leipzig, February 24-26 2012, Hrsg. Jörg Gertel und Anne-Linda A. Augustin, 2-25. Leipzig: Universität Leipzig u. Volkswagenstiftung.

Bank, André. 2009. Die Renaissance des Autoritarismus: Erkenntnisse und Grenzen neuerer Beiträge der Comparative Politics und Nahostforschung. Hamburg Review of Social Sciences 4: 10-41.

Bank, André, und Erik Mohns. 2013. Die syrische Revolte. Protestdynamik, Regimerepression und Internationalisierung. In Arabellions. Zur Vielfalt von Protest und Revolte im Nahen Osten und Nordafrika, Hrsg. Annette Jünemann und Anja Zorob, 85-106. Wiesbaden: Springer VS.

Bank, André, und Thomas Richter. 2013. Autoritäre Monarchien im Nahen Osten. Bedingungen für Überleben und Zusammenbruch seit 1945. In Autokratien im Vergleich. PVS Sonderheft 47, Hrsg. Steffen Kailitz und Patrick Köllner, 284-417. Baden-Baden: Nomos.

Bayat, Asef. 2010. Life As Politics: How Ordinary People Change the Middle East. Stanford: Stanford University Press.

Bayat, Asef. 2013. The Arab Spring and its Surprises. Development and Change 44: 587601.

Beck, Martin, und Simone Hüster. 2013. Politischer Wandel im Nahen Osten. In Arabellions. Zur Vielfalt von Protest und Revolte im Nahen Osten und Nordafrika. Hrsg. Annette Jünemann und Anja Zorob, 197-227. Wiesbaden: Springer VS.

Beinin, Joel, und Frédéric Vairel (Hrsg.). [2010] 2013. Social Movements, Mobilization, and Contestation in the Middle East and North Africa. Stanford: Stanford University Press.

Berman, Sheri. 2013. The Promise of the Arab Spring. In Political Development, no Gain without Pain. Foreign Affairs 92: 64-74.

Bin Talal, El H., und Rolf Schwarz. 2013. The Responsibility to Protect and the Arab World: An Emerging International Norm? Contemporary Security Policy 34: 1-15.

Bouziane, Malika, und Katharina Lenner. 2013. Jenseits ,monarchischer Stabilität‘. Jordanien in Bewegung. In Arabellions. Zur Vielfalt von Protest und Revolte im Nahen Osten und Nordafrika, Hrsg. Annette Jünemann und Anja Zorob, 107-133. Wiesbaden: Springer VS.

Brown, Jonathan. 2011. Salafis and Sufis in Egypt. Washington, D. C.: Carnegie Endowment for International Peace.

Brumberg, Daniel. 2013. Transforming the Arab World's Protection-Racket Politics. Journal of Democracy 24: 88-103.

Brynen, Rex, Pete W. Moore, Bassel F. Salloukh und Marie Joelle Zahar (Hrsg.). 2012. Beyond the Arab Spring. Authoritarianism \& Democratization in the Arab World. Boulder: Lynne Rienner Publishers.

Croissant, Aurel. 2013. Militär und Politik in den arabischen Autokratien. In Friedensgutachten 2013, Hrsg. Marc von Boemcken, Ines-Jacqueline Werkner, Marget Johannsen und Bruno Schoch, 98-111. Berlin: Lit Verlag. 
Czajka, Agnes. 2011. Orientalising the Egyptian Uprising, Take Two: A Response to Rabab el-Mahdi and her Interlocutors. http://www.jadaliyya.com/pages/index/2016/ orientalising-the-egyptian-uprising-take-two_a-res. Zugegriffen: 21.08.2013.

Dabashi, Hamid. 2012. The Arab Spring: The End of Postcolonialism. London/New York: Zed Books.

Dalmasso, Emanuela. 2012. Surfing the Democratic Tsunami in Morocco: Apolitical Society and the Reconfiguration of a Sustainable Authoritarian Regime. Mediterranean Politics 17: 217-232.

Dalmasso, Emanuela, und Francesco Cavatorta. 2013. Democracy, Civil Liberties and the Role of Religion after the Arab Awakening: Constitutional Reforms in Tunisia and Morocco. Mediterranean Politics 18: 225-241.

Davidson, Christopher. 2012. After the Sheikhs: The Coming Collapse of the Gulf Monarchies. London: Hurst.

Dawisha, Adeed. 2013. The Second Arab Awakening. Revolution, Democracy, and the Islamist Challenge from Tunis to Damascus. New York/London: W.W. Norton \& Company.

Del Sarto, Raffaella, und Tobias Schumacher. 2011. From Brussels with Love: Leverage, Benchmarking, and the Action Plans with Jordan and Tunisia in the EU's Democratization Policy. Democratization 18: 932-955.

Demmelhuber, Thomas. 2013. Der Pharao, das Regime und der Staat: Regime- und Elitenwandel nach Mubarak. In Revolution und Regimewandel in Ägypten, Hrsg. Holger Albrecht und Thomas Demmelhuber, 43-61. Baden-Baden: Nomos.

Donker, Teije H. 2013. Re-emerging Islamism in Tunisia: Repositioning Religion in Politics and Society. Mediterranean Politics 18: 207-224.

El-Mahdi, Rabab. 2010. Labour Protests in Egypt: Causes and Meanings. Review of African Political Economy 38: 387-402.

El-Mahdi, Rabab. 2011. Orientalising the Egyptian Uprising. http://www.jadaliyya.com/ pages/index/1214/. Zugegriffen: 21.08.2013.

Frisch, Hillel. 2013. The Egyptian Army and Egypt's 'Spring'. Journal of Strategic Studies 36: $180-204$.

Gause, Gregory. 2011. Why Middle East Studies Missed the Arab Spring. The Myth of Authoritarian Stability. Foreign Affairs 90: 81-90.

Gertel, Jörg, und Rachid Ouaissa. 2014. Urbane Jugendbewegungen. Widerstand und Umbrüche in der arabischen Welt. Bielefeld: transcript.

Grand, Stephen. 2011. Democratization 101: Historical Lessons for the Arab Spring. In The Arab Awakening. America and the Transformation of the Middle East, Hrsg. Kenneth Pollack, 21-28. Washington, D. C.: Brookings Institution Press.

Haas, Mark, und David Lesch. 2012. Introduction. In The Arab Spring. Change and Resistance in the Middle East, Hrsg. Mark Haas und David Lesch, 1-10. Boulder: Westview Press.

Harders, Cilja. 2011. Die Umbrüche in der Arabischen Welt: Zwischen Revolution und Restauration. In Proteste, Revolutionen, Transformationen - die Arabische Welt im Umbruch, Hrsg. Arbeitsstelle Politik des Vorderen Orients, 10-36. Berlin: Freie Universität.

Hoffmann, Anja, und Christoph König. 2013. Scratching the Democratic Facade: Framing Strategies of the 20 February Movement. Mediterranean Politics 18: 1-22. 
Hoffmann, Anja, Malika Bouziane und Cilja Harders. 2013. Analyzing Politics Beyond the Center in an Age of Transformation. In Local Politics and Contemporary Transformations in the Arab World. Governance beyond the Center, Hrsg. Malika Bouziane, Cilja Harders und Anja Hoffmann, 1-21. New York: Palgrave Macmillan.

Hopkins, Nicholas (Hrsg.). 2009. Political and Social Protest in Egypt. Kairo: American University in Cairo Press.

Howard, Philip, und Muzammil M. Hussain (Hrsg.). 2013. Democracy's Fourth Wave? Digital Media and the Arab Spring: Oxford/New York: Oxford University Press.

Hudson, Michael. 2011. Awakening, Cataclysm, or Just a Series of Events? Reflections on the Current Wave of Protest in the Arab World. http://www.jadaliyya.com/pages/ index/1601/awakening-cataclysm-or-just-a-series-of-events-ref. Zugegriffen: 21.08.2013.

Hüllen, Vera van. 2012. The European Union and Democracy Promotion in the Mediterranean. Strategic Choices after the Arab Spring. In Policy Change in the EU's Immediate Neighbourhood. A Sectoral Approach, Hrsg. Tanja A. Börzel und Katrin Böttger, 119-144. Baden-Baden: Nomos.

Huntington, Samuel. 1968. Political Order in Changing Societies. New Haven: Yale University Press.

Khouri, Rami. 2011. Drop the Orientalist Term “Arab Spring”. The Daily Star Lebanon. http:// www.dailystar.com.lb/Article.aspx?id=146410\#axzz2nuch9IRa. Zugegriffen: 25.11.2013.

Kodmani, Bassma. 2010. Democratization by whom? Resistance to Democracy Promotion in the Middle East. In New Challenges to Democratization, Hrsg. Peter Burnell und Richard Youngs, 153-170. London/New York: Routledge.

Koselleck, Reinhart. 2006. Begriffsgeschichten. Studien zur Semantik und Pragmatik der politischen und sozialen Sprache. Frankfurt a. M.: Suhrkamp.

Lang, Anthony. 2013. From Revolutions to Constitutions: the Case of Egypt. International Affairs 89: 345-363.

Leenders, Reinoud, und Steven Heydemann. 2012. Popular Mobilization in Syria: Opportunity and Threat, and the Social Networks of the Early Risers. Mediterranean Politics 17: 139-159.

Lobe, Adrian. 2011. Politologie. Die Wissenschaft vom stabilen Orient. Frankfurter Allgemeine Zeitung. http://www.faz.net/aktuell/feuilleton/geisteswissenschaften/politologiedie-wissenschaft-vom-stabilen-orient-1606776.html. Zugegriffen: 25.11.2013.

Lübben, Ivesa. 2013. Auf dem Weg zum Gottesstaat? Zur politischen Partizipation von Islamisten am Beispiel Ägyptens nach Mubarak. In Der Arabische Frühling: Hintergründe und Analysen, Hrsg. Thorsten G. Schneiders, 163-180. Wiesbaden: Springer VS.

Miles, Hugh. 2011. The Al Jazeera Effect. The Inside Story of Egypt's TV Wars and How Saudi Arabia Could Be Next. Foreign Policy. http://www.foreignpolicy.com/articles/2011/02/08/the_al_jazeera_effect. Zugegriffen: 25.11.2013.

Moghadam, Valentine. 2013. What is Democracy? Promises and Perils of the Arab Spring. Current Sociology 61: 393-408.

Ouaissa, Rachid. 2013. Blockierte Mittelschichten als Motor der Veränderungen in der arabischen Welt? In Arabellions. Zur Vielfalt von Protest und Revolte im Naben Osten und Nordafrika, Hrsg. Annette Jünemann und Anja Zorob, 257-276. Wiesbaden: Springer VS.

Pace, Michelle, und Francesco Cavatorta. 2012. The Arab Uprisings in Theoretical Perspective - An Introduction. Mediterranean Politics 17: 125-138. 
Perthes, Volker. 2013. Arabische Turbulenzen. Blätter für Internationale Politik 2: 43-51.

Pollack, Kenneth (Hrsg.). 2011. The Arab Awakening. America and the Transformation of the Middle East. Washington, D. C.: Brookings Institution Press.

Preysing, Domenica. 2013. Tunesien: Vorreiter des Aufbruchs, Vorbild des Wandels? In Arabellions. Zur Vielfalt von Protest und Revolte im Nahen Osten und Nordafrika, Hrsg. Annette Jünemann und Anja Zorob, 43-66. Wiesbaden: Springer VS.

Ramadan, Tariq. 2012. The Arab Awakening: Islam and the New Middle East. London: Allen Lane.

Rebhan, Helga. 1986. Geschichte und Funktion einiger politischer Termini im Arabischen des 19. Jahrhunderts (1798-1882). Wiesbaden: Harrassowitz.

Richter, Carola. 2013. Liberalisierungsprozesse im ägyptischen Mediensystem und ihre Bedeutung für den politischen Umbruch 2011. In Revolution und Regimewandel in Ägypten, Hrsg. Holger Albrecht und Thomas Demmelhuber, 143-162. Baden-Baden: Nomos.

Schlumberger, Oliver. 2011. Kritik an Politikwissenschaft. Nahost-Experten, echte und andere. Frankfurter Allgemeine Zeitung. http://www.faz.net/aktuell/feuilleton/forschungund-lehre/kritik-an-politikwissenschaft-nahost-experten-echte-und-andere-1610582. html. Zugegriffen: 25.11.2013.

Schlumberger, Oliver, Nadine Kreitmeyer und Thorsten Matzke. 2013. Arabische Revolten und politische Herrschaft: Wie überlebensfähig sind Autokratien im Vorderen Orient? In Der Arabische Frühling: Hintergründe und Analysen, Hrsg. Thorsten G. Schneiders, 33-63. Wiesbaden: Springer VS.

Schumacher, Tobias. 2012. New Neighbors, Old Formulas? The ENP One Year after the Start of the Arab Spring. In The Arab Spring: One Year After. Transformation Dynamics, Prospects of Democratization and the Future of Arab-European Cooperation, Hrsg. Amine Ghali, 87-104. Gütersloh: Bertelsmann Stiftung.

Schumann, Christoph. 2013. Revolution oder Revolte? Arabischer Frühling jenseits des Autoritarismus- und Transitionsparadigmas. In Revolution und Regimewandel in Ägypten, Hrsg. Holger Albrecht und Thomas Demmelhuber, 19-40. Baden-Baden: Nomos.

Seib, Philip. 2008. The Al Jazeera Effect: How the New Global Media are Reshaping World Politics. Washington, D.C.: Potomac Books.

Shihade, Magid. 2012. On the Difficulty in Predicting and Understanding the Arab Spring: Orientalism, Euro-Centrism, and Modernity. International Journal of Peace Studies 17: 57-70.

Springborg, Robert. 2011. Economic Involvement of Militaries. Roundtable. International Journal of Middle East Studies 43: 397-399.

Stepan, Alfred, und Juan Linz. 2013. Democratization Theory and the "Arab Spring”. Journal of Democracy 24: 15-30.

Teti, Andrea. 2012a. Beyond Lies the Wub: Challenges of (Post)Democratization. Middle East Critique 21: 5-24.

Teti, Andrea. 2012b. The EU's First Response to the 'Arab Spring': A Critical Discourse Analysis of the Partnership for Demcoracy and Shared Prosperity. Mediterranean Politics 17: 266-284.

Tömmel, Ingeborg. 2013. The New Neighborhood Policy of the EU: An Appropriate Response to the Arab Spring? Democracy and Security 9: 19-39. 
Wallstreet Journal. 7. Juli 2013. http://online.wsj.com/news/articles/SB100014241278873 24399404578583932317286550. Zugegriffen: 25.11.2013.

Weipert-Fenner, Irene. 2013. Wegbereiter oder treibende Kraft? Die Rolle der Arbeiter in der ägyptischen Revolution. In Revolution und Regimewandel in Ägypten, Hrsg. Holger Albrecht und Thomas Demmelhuber, 209-231. Baden-Baden: Nomos.

Weipert-Fenner, Irene. 2014. Parliamentary Protest against Mubarak, 2005-2010. In Routledge Handbook of the Arab Spring. Rethinking Democratization, Hrsg. Larbi Sadiki. London: Routledge (im Erscheinen).

Wirth, Ruth. 2013. Zehn Millionen Stimmen: Wen wäblen Ägyptens Sufis? GIGA Focus Nahost 2. http://www.giga-hamburg.de/en/system/files/publications/gf_nahost_1302. pdf. Zugegriffen: 25.11.2013.

Yom, Sean. 2012. Understanding the Resilience of Monarchy during the Arab Spring. Foreign Policy Research Institute. http:/www.fpri.org/enotes/2012/201204.yom.monarchyarab-spring.html. Zugegriffen: 25.11.2013.

Zeghal, Malika. 2013. Competing Ways of Life: Islamism, Secularism, and Public Order in the Tunisian Transition. Constellations 20: 254-274.

Žižek, Slavoj. 2012. The Year of Dreaming Dangerously. London/New York: Verso.

Zorob, Anja. 2013. Der Zusammenbruch des autoritären Gesellschaftsvertrags. Sozioökonomische Hintergründe der arabischen Proteste. In Arabellions. Zur Vielfalt von Protest und Revolte im Nahen Osten und Nordafrika, Hrsg. Annette Jünemann und Anja Zorob, 229-256. Wiesbaden: Springer VS.

\section{Autorenangaben}

Dr. des. Irene Weipert-Fenner,

Forschungsnetzwerk „Re-Konfigurationen. Geschichte, Erinnerung und Transformationsprozesse im Mittleren Osten und Nordafrika “, Universität Marburg,

Deutschhausstr. 3,

35032 Marburg,

Email: irene.weipertfenner@uni-marburg.de 